\title{
End Group-functionalization and Synthesis of Block- copolythiophenes by Modified Nickel Initiators
}

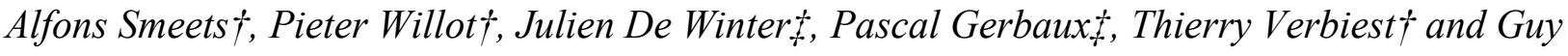 \\ Koeckelberghst*
}

$†$ Laboratory of Molecular Electronics and Photonics, Katholieke Universiteit Leuven,

Celestijnenlaan 200F, B-3001 Heverlee, Belgium, and \$Mass Spectrometry Research Group, Interdisciplinary Center for Mass Spectrometry, University of Mons- UMONS, 20 Place du Parc, B7000 Mons, Belgium

guy.koeckelberghs@chem.kuleuven.be

\section{RECEIVED DATE (to be automatically inserted after your manuscript is accepted if required according to the journal that you are submitting your paper to)}

Polythiophenes with alcohol, tosylate, azide, ethynylene, carboxylic acid and amine end groups were prepared by a combination of functionalized nickel initiators and post-polymerization reactions. The azide and ethynylene polymers were subsequently used in a click reaction to produce a conjugated block-copolymer. Finally, a conjugated triblock-copolymer was synthesized by means of a chain growth polymerization initiated by a binuclear nickel initiator.

KEYWORDS $\pi$-conjugated polymers, block-copolymers, nickel initiators, functional end groups, polythiophenes

\section{Introduction}

Conjugated polymers (CPs) remain intensively studied materials because they hold promise as active layers in applications in the field of molecular electronics. ${ }^{1}$ A major break-through was 
achieved by the groups of McCullough ${ }^{2-3}$ and Yokozawa, ${ }^{4-5}$ who demonstrated that the $\mathrm{Ni}(\mathrm{dppp}) \mathrm{Cl}_{2^{-}}$ initiated $(\mathrm{dppp}=$ 1,3-bisdiphenylphosphinopropane) polymerization of poly(3-hexylthiophene) (P3HT) proceeds via a controlled chain-growth mechanism. This allows the synthesis of welldefined polythiophenes with predicted molar mass and narrow polydispersities. Moreover, the controlled nature of the polymerization can also be exploited to end-cap the polymer chains, introducing functional groups, which can, after some transformation, act as initiators for a controlled radical polymerization of a second block. In this way, block-copolymers composed of a P3HT block and a non-conjugated block (e.g. PMMA, PS,...) can be prepared. ${ }^{6-8}$ Alternatively, the living nature can be used to prepare all-conjugated block copolymers by successive addition of the respective monomers. In this way, the synthesis of block-copolymers composed of two different P3AT blocks, ${ }^{9-}$ ${ }^{14}$ a P3AT and a poly(3-alkoxythiophene) (P3AOT) block, ${ }^{15-16}$, a poly(selenophene) block ${ }^{17}$ or a poly(fluorene) block $^{18}$ and a poly(pyrrole)-block-poly(phenylene $)^{19}$ have been achieved.

End-capping of polymers chains requires a living nature of the polymerization. This is indeed the case for P3ATs and it has also been found for some other conjugated polymers (to a certain extent) using either Ni- or Pd-catalysts. ${ }^{20-23}$ Nevertheless, some other CPs polymerize via an albeit chaingrowth, but not-living, mechanism. However, if the mechanism of the $\mathrm{Ni}(\mathrm{dppp}) \mathrm{Cl}_{2}$-initiated polymerization is examined into more detail, it becomes clear that a chloro organonickel(dppp) species can be considered as the actual initiator. More specifically, an arylmagnesium moiety performs a nucleophilic attack on $\mathrm{Ni}(\mathrm{dppp}) \mathrm{Cl}_{2}$, forming the chloro organonickel(dppp) initiator, which then reacts with more monomer, starting the propagation cycle. As a consequence, a functional group can also conveniently be introduced in the beginning of each polymer chain by initiating the polymerization from such organonickel species equipped with the desired functional group. Several methodologies have been reported for the synthesis of such molecules and their use as initiators for the P3HT polymerization. Luscombe and coworkers prepared aryl-Ni complexes from (in situ prepared) $\mathrm{Ni}\left(\mathrm{PPh}_{3}\right)_{4}$ or $\mathrm{Ni}(\mathrm{cod})_{2}$ and the corresponding aryl bromides or chlorides. ${ }^{24-26}$ Kiriy reacted aryl bromides with either $\mathrm{Ni}\left(\mathrm{PPh}_{3}\right)_{4}$ or diethyl(bipyridyl)nickel, followed by a ligand exchange with dppp. ${ }^{27-28} \mathrm{~A}$ Ni-initiator capable of initiating a NMP was also prepared through this 
methodology. ${ }^{29}$ We prepared Ni-initiators bearing a (protected) alcohol or acetylenic group by reacting $\mathrm{Ni}\left(\mathrm{PPh}_{3}\right)_{4}$ with the appropriate aryl bromide and used them to polymerize P3HT, P3OATs, poly(3-alkylsulfanylthiophene)s and poly(phenylene)s. ${ }^{30}$

In this manuscript, we first expand the latter protocol by synthesizing a broad variety of functional Ni initiators, offering the possibility to prepare conjugated polymers equipped with alcohol, tosylate, azide, amine, ethynyl and carboxylic acid functional groups. Next, the click reaction was used to couple two different conjugated polymers (in casu P3AT and P3AOT) resulting in an all-conjugated block copolymer with a non-conjugated spacer. Finally, we prepared a bifunctional Ni-initiator and used it to initiate a P3HT polymerization. Moreover, the living nature of this polymerization could be exploited for the synthesis of a P3AOT-block-P3HT-block-P3AOT triblock-copolymer.

\section{Experimental}

\section{Reagents and Instrumentation}

All reagents were purchased from Sigma Aldrich, Acros Organics, Merck, Wako, ABCR and Alfa Aesar. Reagent grade solvents were dried by distillation over a suitable drying agent. Bromo-[2methyl-4-(trimethylsilylethynylene)phenyl]bis(triphenylphosphine)nickel ${ }^{30}$

(1) and 2magnesiochloro-4-hexyl-5-bromothiophene ${ }^{4}$ was prepared according to literature procedures. Before each polymerization, a small aliquot of 2-magnesiochloro-4-hexyl-5-bromothiophene was quenched with $\mathrm{D}_{2} \mathrm{O}$ and analyzed by ${ }^{1} \mathrm{H}-\mathrm{NMR}$ to verify its conversion. Gel Permeation chromatography (GPC) measurements were done with a Shimadzu 10A apparatus with a tunable absorbance detector and a differential refractometer in tetrahydrofuran as eluent toward polystyrene standards. Mass spectra were recorded using an Agilent HP5989. ${ }^{1} \mathrm{H}$ nuclear magnetic resonance ( $\left.{ }^{1} \mathrm{H}-\mathrm{NMR}\right)$ measurements were carried out with a Bruker Avance $300 \mathrm{MHz}, 400 \mathrm{MHz}$ and $600 \mathrm{MHz} .{ }^{31} \mathrm{P}-\mathrm{NMR}$ measurements were carried out with a Bruker Avance $400 \mathrm{MHz}$. MALDI-ToF mass spectra were recorded using a Waters QToF Premier mass spectrometer equipped with a nitrogen laser, operating at $337 \mathrm{~nm}$ with a maximum output of $500 \mathrm{~J} / \mathrm{m}^{2}$ delivered to the sample in 4 ns pulses at $20 \mathrm{~Hz}$ repeating rate. Time-offlight mass analyses were performed in the reflection mode at a resolution of about 10000 . The matrix, trans-2-[3-(4-t-Butyl-phenyl)-2-methyl-2-propenylidene]malononitrile $\quad$ (DCTB), was 
prepared as $20 \mathrm{mg} / \mathrm{mL}$ solution in chloroform. The matrix solution $(1 \mu \mathrm{L})$ was applied to a stainless steel target and air dried. Polymer samples were dissolved in chloroform to obtain $1 \mathrm{mg} / \mathrm{mL}$ solutions. $1 \mu \mathrm{L}$ aliquots of these solutions were applied onto the target area already bearing the matrix crystals, and then air dried. IR spectra were recorded using a Bruker Alpha-p apparatus in ATR mode.

\section{Synthesis of the bifunctional initiator}

\section{Synthesis of 2-bromo-3-methylthiophene (16)}

N-Bromosuccinimide (50.0 mmol, $8.88 \mathrm{~g}$ ) was added portion wise to a solution of 3methylthiophene (15) $(50.0 \mathrm{mmol}, 4.91 \mathrm{~g})$ in $\mathrm{CHCl}_{3}(20 \mathrm{~mL})$ and $\mathrm{AcOH}(20 \mathrm{~mL})$, which had been cooled to $0^{\circ} \mathrm{C}$, shielded from light and was equipped with a $\mathrm{CaCl}_{2}$ drying tube. The reaction was then allowed to reach room temperature overnight, after which aqueous $\mathrm{NaHSO}_{3}$ and $\mathrm{NaHCO}_{3}$ were added. The mixture was then extracted with hexane, the organic layer was dried over $\mathrm{MgSO}_{4}$ and the solvent removed in vacuo. The crude product was then vacuum distilled $\left(66^{\circ} \mathrm{C}, 16 \mathrm{mmHg}\right)$ and the pure product was isolated as a light yellow oil.

Yield $=7.40 \mathrm{~g}(83 \%)$

${ }^{1} \mathrm{H}-\mathrm{NMR}\left(\mathrm{CDCl}_{3}\right): 7.16(\mathrm{~d}, 1 \mathrm{H}, 5.5 \mathrm{~Hz}), 6.77$ (d, $\left.1 \mathrm{H}, 5.5 \mathrm{~Hz}\right), 2.20$ (s, $\left.3 \mathrm{H}\right)$

\section{Synthesis of 5,5'-dibromo-4,4'-dimethyl-2,2'-bithiophene (17)}

A solution of 16 (20.0 mmol, $3.54 \mathrm{~g})$ in dry DMSO (40 mL) was purged with argon and added to a solution of $\mathrm{PdCl}_{2}(\mathrm{PhCN})_{2}(0.600 \mathrm{mmol}, 0.230 \mathrm{~g}), \mathrm{AgNO}_{3}(40.0 \mathrm{mmol}, 6.79 \mathrm{~g})$ and $\mathrm{KF}(40.0 \mathrm{mmol}$, $2.32 \mathrm{~g})$ in dry DMSO $(40 \mathrm{~mL})$. The mixture was allowed to react for $3 \mathrm{~h}$ at $60^{\circ} \mathrm{C}$, after which hexane was added. The resulting precipitate was then removed by filtration and subsequently washed with hexane and diethylether. The organic layer was then washed with a $\mathrm{NaCl}$ solution, dried over $\mathrm{MgSO}_{4}$ and the solvent removed in vacuo. Finally, the crude product was purified by column chromatography $\left(\mathrm{SiO}_{2}\right.$, eluent: hexane) and isolated as a white solid.

Yield: $2.02 \mathrm{~g}(57 \%)$

${ }^{1} \mathrm{H}-\mathrm{NMR}\left(\mathrm{CDCl}_{3}\right): \delta=6.76(\mathrm{~s}, 2 \mathrm{H}), 2.17(\mathrm{~s}, 6 \mathrm{H})$

\section{Synthesis of 18}


A solution of $17(2.00 \mathrm{mmol}, 0.704 \mathrm{~g})$ in dry toluene $(50 \mathrm{ml})$ was added under argon atmosphere to $\mathrm{Ni}\left(\mathrm{PPh}_{3}\right)_{4}(5.00 \mathrm{mmol}, 5.54 \mathrm{~g})$. The reaction was allowed to proceed overnight, after which toluene $(50 \mathrm{~mL})$ was added and the solution was filtered. The supernatant was concentrated in vacuo and the product precipitated in $\mathrm{MeOH}$, yielding a yellow-brown powder.

Yield: $0.125 \mathrm{~g}(4 \%)$

${ }^{1} \mathrm{H}-\mathrm{NMR}\left(\mathrm{CDCl}_{3}\right): \delta=7.61(\mathrm{~m}, 24 \mathrm{H}), 7.25(\mathrm{~m}, 36 \mathrm{H}), 5.62(\mathrm{~s}, 2 \mathrm{H}), 1.47(\mathrm{~s}, 6 \mathrm{H})$

\section{Synthesis of block-copolymer using the click reaction Synthesis of P3HT-2-P3AOT}

An argon purged solution of PMDTA $(5.60 \mu \mathrm{mol}, 0.97 \mathrm{mg})$ in dry THF $(5 \mathrm{~mL})$ was added to a suspension of $\mathrm{CuBr}(4.51 \mu \mathrm{mol}, 0.650 \mathrm{mg})$ and dry THF $(5 \mathrm{~mL})$. A solution of P3HT-2-N 3 (68.7 $\mu \mathrm{mol}, 11.4 \mathrm{mg})$ and P3AOT-E $(126 \mu \mathrm{mol}, 30.0 \mathrm{mg})$ in dry THF $(50 \mathrm{~mL})$ was added to this solution and the reaction mixture was stirred overnight under an argon atmosphere. Then, chloroform and an aqueous solution of $\mathrm{NH}_{3}$ were added and the mixture was extracted. The organic layer was dried over $\mathrm{Na}_{2} \mathrm{SO}_{4}$ and concentrated to a volume of approximately $15 \mathrm{~mL}$, precipitated in methanol and filtered off. The polymer was further purified by means of Soxhlet extraction with pentane, hexane, heptane and finally with chloroform. The chloroform solution was precipitated in $\mathrm{MeOH}$, filtered off and dried in vacuo.

Yield $=18.9 \mathrm{mg}(48 \%)$

\section{Synthesis of triblock-copolymer}

An argon purged mixture of $18(0.0486 \mathrm{mmol}, 0.0740 \mathrm{~g})$ and dppp $(0.107 \mathrm{mmol}, 0.0440 \mathrm{~g})$ in dry THF $(6.5 \mathrm{ml})$ was stirred for $30 \mathrm{~min}$ at room temperature. In a next step, 2-magnesiochloro-4-hexyl5-bromothiophene $(0.800 \mathrm{mmol})$ in dry THF $(5.5 \mathrm{~mL})$ was added to the initiator solution and stirred for $1 \mathrm{~h}$ at room temperature. Next, $3.8 \mathrm{~mL}$ of the mixture was quenched with $\mathrm{HCl}$, precipitated in $\mathrm{MeOH}$, filtered off and dried in vacuo. Washing with acetone yielded P3HT-bth-P3HT as a dark purple solid. To the remaining solution $(8.2 \mathrm{~mL})$, 2-bromomagnesio-5-bromo-3-(3,7- $(S)-$ dimethyloctyloxy)thiophene (1.65 mmol) (prepared as for P3AOT-TMS) in dry THF (12 mL) was added and the mixture was allowed the react overnight. The polymer was then precipitated in 
$\mathrm{MeOH}$, further purified by means of Soxhlet extraction with pentane, hexane, heptane and finally with THF. The THF solution was precipitated in $\mathrm{MeOH}$, filtered off and dried in vacuo, yielding P3AOT-P3HT-P3AOT as a black solid.

Yield $($ P3HT-bth-P3HT) $=26.5 \mathrm{mg}(64 \%)$

Yield $($ P3AOT-P3HT-P3AOT $)=185 \mathrm{mg}(32 \%$ based on P3AOT $)$

\section{Results and discussion}

\section{Initiator design}

In a previous communication, ${ }^{30}$ we established a protocol for the synthesis of arylnickel complexes and their subsequent application as functional initiators for the synthesis of conjugated polymers (CP). This allowed us to insert end groups into CPs even if their polymerization is not controlled. We decided to expand upon this protocol by inserting a wider variety of functional groups, which could then be employed in post-polymerization reactions. The end groups in question should allow straightforward coupling of the $\mathrm{CP}$ to the desirable substrates, i.e. other polymers or nanoparticles. Two candidates which immediately come to mind are azide and ethynylene functionalities, as they can be employed in so called CuAAC click chemistry, which is robust and can be achieved using mild conditions, ${ }^{31-32}$ fulfilling the requirement stated earlier. Two other functional groups we deemed worth pursuing are amine and carboxylic functionalities, because they could be useful for coupling CPs with nanoparticles ${ }^{33}$ or surfaces. ${ }^{34}$ As for the choice of the CPs, we decided to use poly(3hexylthiophene) (P3HT) as main $\mathrm{CP}$, because the polymerization of P3HT with functionalized nickel initiators yields polymers with a high degree of functionalization. ${ }^{30}$ As second polymer we decided to use poly(3-(3,7-(S)-dimethyloctyloxy)thiophene (P3AOT). Although the polymerization of P3AOT is less well-behaved and its functionalization usually incomplete, ${ }^{30,35}$ it is still a worthwhile choice because of its substantially different physiochemical properties compared to P3HT, which should allow for easy discrimination of both CPs using standard spectroscopic techniques. For the reasons mentioned above, we decided to synthesize P3HT with azide, amine and carboxylic end groups, while a P3AOT would be synthesized with an ethynylene end group. 
In order to synthesize both CPs with the desired end groups, the corresponding nickel initiators were synthesized from $\mathrm{Ni}\left(\mathrm{PPh}_{3}\right)_{4}$ and the appropriate arylbromide. Although all four functional end groups should not pose any problems in this respect, they are unfortunately incompatible with the polymerization conditions of both $\mathrm{P} 3 \mathrm{HT}$ and $\mathrm{P} 3 \mathrm{AOT}$, because both polymerizations proceed through the nickel catalyzed coupling of an organomagnesium (or organozinc) monomer. The end groups in question thus had to be either protected or formed after polymerization from a precursor moiety. To ethynylene, amine and carboxylic functional groups, the former applies, as well established methods for protection (against either organomagnesium or organozinc compounds) are available. The azide functionality, however, has to be formed through post-polymerization reactions of a precursor end group, as no direct method of protection is available. As precursor end group, we selected an alcohol functionality, because of its easy protection, deprotection and synthetic versatility.

The synthesis of the protected acetylene-functionalized initiator, bromo-[2-methyl-4[(trimethylsilyl)ethynyl]phenyl]bis(triphenylphosphine)nickel(II) (1) has been described previously. ${ }^{25}$ For the (protected) alcohol-functionalized initiator (5), 4-bromo-3-methylphenol (2) was alkylated with ethylene carbonate in DMF using $\mathrm{K}_{2} \mathrm{CO}_{3}$ as base, forming $\mathbf{3}$. Subsequently, 3 was protected using TBDMSCl and imidazole in DMF, yielding 1-bromo-2-methyl-4-[2-( $t$ butyldimethylsilyloxy)ethyloxy] benzene (4). Reaction of 4 with $\mathrm{Ni}\left(\mathrm{PPh}_{3}\right)_{4}$ resulted in the formation of bromo[2-methyl-4-[2-(t-butyldimethylsilyloxy)ethyloxy]phenyl]bis(triphenylphopshine)nickel(II) (5). In order to introduce carboxyl groups in the polymers, we first selected an oxazoline moiety as a protecting group compatible with the organomagnesium monomer. The corresponding nickel initiator could be prepared by converting 4-bromo-3-methylbenzoic acid (6) to its acid chloride (7) by means of oxalyl chloride, which was reacted with 2-amino-2-methyl-1-propanol and $\mathrm{SOCl}_{2}$, yielding 2-(4-bromo-3-methylphenyl)-4,5-dihydro-4,4-dimethyloxazole (8). Analogous to the synthesis of previous initiators, 8 was then reacted with $\mathrm{Ni}\left(\mathrm{PPh}_{3}\right)_{4}$, forming the nickel initiator 9. However, after polymerization, it turned out that the carboxylic acid could not be deprotected without degradation of the polymer. Indeed, treatment with $\mathrm{KOH}$ in THF did not result in any reaction, while treatment with aqueous $\mathrm{HCl}$ (either in dioxane or chloroform) or $\mathrm{MeI}$ and 
subsequently $\mathrm{NaOH}$ resulted in degradation reactions. Because of our inability to convert the oxazoline end group back to the carboxylic acid, we were forced to consider a different protecting group. We decided to protect the carboxylic acid by esterificating it with $t$-butanol.

In order to synthesize the $\mathrm{COO} t \mathrm{Bu}$ functionalized initiator, 7 was reacted with $\mathrm{KO} t \mathrm{Bu}$, yielding $t$ butyl(4-bromo-3-methyl)benzoate (10). Reaction of $\mathbf{1 0}$ with $\mathrm{Ni}\left(\mathrm{PPh}_{3}\right)_{4}$ yielded the $\mathrm{COO} t \mathrm{Bu}$ functionalized initiator (11).

The introduction of an amine group into the polymers required the synthesis of a nickel initiator with an amine functionality, which also needs to be protected against the organomagnesium monomer. At first we decided to employ STABASE protection (STABASE $=1,1,4,4$ tetramethyldisilyl azacyclopentane) for the amine. However, we were unable to isolate the necessary STABASE-protected precursor for the nickel initiator as it spontaneously decomposed back to the free amine during purification. We therefore had to select an alternative and switched to Boc protection instead. First, we synthesized 2-(4-bromo-3-methylphenyloxy)ethylamine (12) by performing a Mitsunobu reaction of $\mathbf{3}$ with phthalimide, subsequent hydrazinolysis of the phthalimide intermediate yielded the primary amine $\mathbf{1 2}$ after workup. The amine $\mathbf{1 2}$ was then protected using $\mathrm{Boc}_{2} \mathrm{O}$ after which ${ }^{1} \mathrm{H}-\mathrm{NMR}$ and MS analysis indicated that the amine had been bisprotected to form 13. Finally, reaction of 13 with $\mathrm{Ni}\left(\mathrm{PPh}_{3}\right)_{4}$ yielded the corresponding nickel initiator 14. 

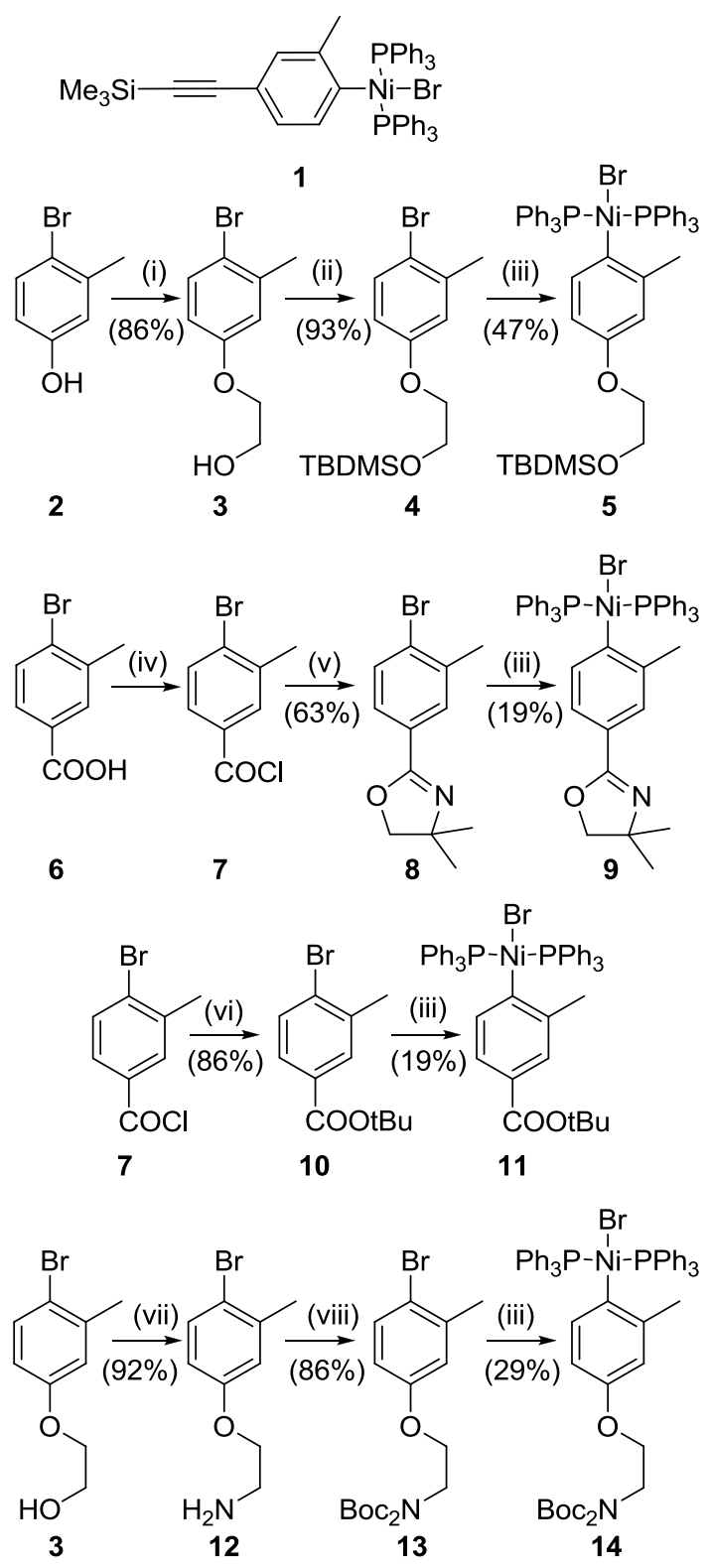

Scheme 1. Synthesis of the Ni-initiators. (i) ethylene carbonate, $\mathrm{K}_{2} \mathrm{CO}_{3}$ (ii) TBDMSCl, imidazole (iii) $\mathrm{Ni}\left(\mathrm{PPh}_{3}\right)_{4}$ (iv) oxalyl chloride, $\mathrm{DMF}$ (v) 2-amino-2-methyl-1-propanol, $\mathrm{SOCl}_{2}$ (vi) $\mathrm{KO} t \mathrm{Bu}$ (vii) 1) DIAD, phthalimide 2) $\mathrm{PPh}_{3}, \mathrm{~N}_{2} \mathrm{H}_{2}$ (viii) $\mathrm{Boc}_{2} \mathrm{O}$, DMAP

\section{Synthesis of the polymers}

The polymers were prepared by a Ni(dppp)-mediated polymerization of metallated thiophenes, as depicted in scheme 2. In the case of P3AOT, the actual monomer, 2-bromomagnesio-5-bromo-3(3,7-(S)-dimethyloctyloxy)thiophene, was prepared in situ from $\mathrm{MgBr}_{2}$ and 2-lithio-5-bromo-3-(3,7(S)-dimethyloctyloxy)thiophene, which was in turn prepared from 2-bromo-3-(3,7-(S)dimethyloctyloxy)thiophene and $\mathrm{LDA}^{35}$ The monomer in the P3HT polymerization is 2- 
magnesiochloro-4-hexyl-5-bromothiophene which was prepared from 2-bromo-5-iodo-3hexylthiophene and $i \operatorname{PrMgCl}$. The $\mathrm{COO} t \mathrm{Bu}$ and $\mathrm{Boc}_{2} \mathrm{~N}$ moieties are not compatible with organomagnesium reagents and thus polymerization conditions which use organomagnesium monomers cannot be employed. Fortunately, the synthesis of P3HT can also be accomplished by an organozinc monomer ${ }^{3,36}$ using the same catalyst and conditions, only transmetalation of the organomagnesium monomer with $\mathrm{ZnBr}_{2}$ is required. Because the Ni-catalyzed polymerization of P3HT is controlled when dppp is used as ligand, ${ }^{25,37}$ ligand exchange was first performed in situ before the monomers were added, thus forming the actual initiator. In order to verify whether the ligand exchange indeed takes place (quantitatively), we monitored this reaction in a separate experiment by ${ }^{31} \mathrm{P}-\mathrm{NMR}$ spectroscopy for all initiators. After $30 \mathrm{~min}$, the sole species detected in all cases except for 1 were liberated $\mathrm{PPh}_{3}$, excess dppp ( 2 equivalents were used) and the end-product (Figure 1). The apparent broadness of the peaks can be attributed to the fact that is actually a (less resolved) doublet. No starting product, nor degradation compounds (e.g. Ni(dppp) $\left.)_{2}\right)$ were found (see SI, Figures S23-26). For all P3HTs, $\left[\mathrm{M}_{0}\right] /[\mathrm{In}]=15$.

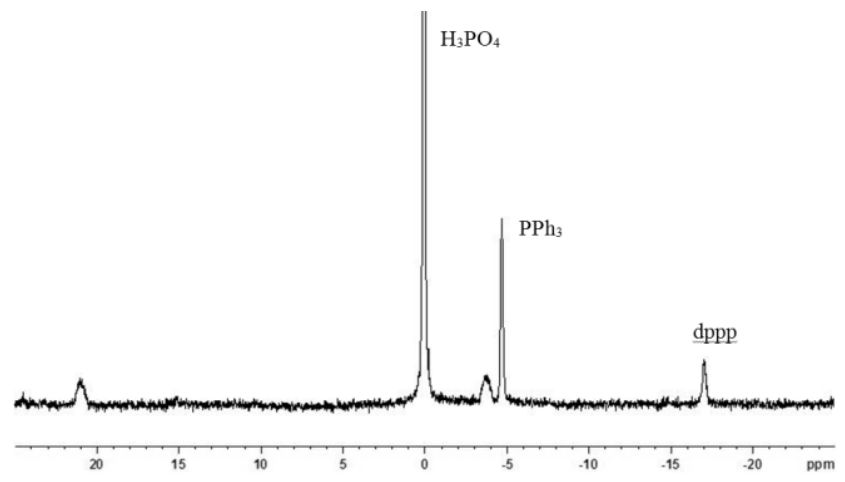

Figure 1. ${ }^{31} \mathrm{P}-\mathrm{NMR}$ spectrum of $5(6.70 \mu \mathrm{mol})$ after $30 \mathrm{~min}$ of ligand exchange with dppp (13.3 $\mu \mathrm{mol})$ in THF $(0.5 \mathrm{~mL})$.

Thus, the synthesis of the ethynylene-functionalized P3AOT was accomplished by first performing ligand exchange on $\mathbf{1}$ with dppp, after which this mixture was used to initiate the polymerization of 2-bromomagnesio-5-bromo-3-(3,7-(S)-dimethyloctyloxy)thiophene, yielding P3AOT-TMS. MALDI-ToF analysis (see Figure S37) of P3AOT-TMS revealed that the main end groups are $\mathrm{Br} / \mathrm{TMS}$, with some minor $\mathrm{H} / \mathrm{Br}$ content. This is the expected result, as the 
polymerization of poly(3-alkoxythiophenes) is not controlled, ${ }^{35}$ yielding polymer chains in which chain end degradation of the polymer-Ni complex occurred. Signals corresponding to the loss of a $\mathrm{C}_{10} \mathrm{H}_{21} \cdot$ radical are also detected in the mass spectrum and were previously ascribed to an aromatic $\beta$-cleavage from the ionized polymer chain. ${ }^{38}$

In order to prepare P3HT with an alcohol functionality, 5 was first reacted with dppp, after which a solution of 2-magnesiochloro-4-hexyl-5-bromothiophene was added, resulting in the formation of P3HT-2-OTBDMS. The presence of the ethyl-OTBDMS moiety was confirmed by ${ }^{1} \mathrm{H}-\mathrm{NMR}$, with the ethyl protons giving rise to two triplets, respectively at 4.19 and $3.61 \mathrm{ppm}$. Also, MALDI-ToF analysis indicates that the majority of the P3HT is OTBDMS/H terminated with some remainder $\mathrm{H} / \mathrm{H}$ terminated P3HT (see Figure $\mathrm{S} 38) . \mathrm{H} / \mathrm{H}$ terminated polymers require $\mathrm{Ni}(\mathrm{dppp}) \mathrm{X}_{2}(\mathrm{X}=\mathrm{Cl}$ or Br) as initiator. Since the ${ }^{31} \mathrm{P}-\mathrm{NMR}$ spectra indicate the absence of such species at the moment the monomer is introduced, such Ni-complex must be formed when the monomer is added. The presence of non-functionalized P3HT is not necessarily detrimental, as it will simply not react during postpolymerization reactions (coupling reactions, block-copolymer formation, ...) and can thereafter be removed by fractionation.

For the polymerization of the carboxyl-functionalized P3HT, 2-magnesiochloro-4-hexyl-5bromothiophene was first transmetallated with $\mathrm{ZnBr}_{2}$ to 2-bromozincio-4-hexyl-5-bromothiophene. Then, 11 was allowed to undergo ligand exchange with dppp and used to initiate the polymerization of the 2-bromozincio-4-hexyl-5-bromothiophene, resulting in the formation of P3HT-COOtBu. The MALDI-ToF analysis (See Figure S39) confirmed that the polymerization reaction is quantitatively initiated by 11. Decomposition reactions, i.e. losses of isobutene $(56 \mathrm{u})$ from the ionized oligomers, were also detected and are characteristic of the COOtBu moiety. Surprisingly, the dominant ion series did not correspond to the $t \mathrm{Bu} / \mathrm{H}$ end groups, but to $t \mathrm{Bu} / \mathrm{C}_{4} \mathrm{H}_{7} \mathrm{O}$ end groups. This composition was unambiguously demonstrated by an accurate mass measurement performed on ionized $\mathrm{C}_{4} \mathrm{H}_{7} \mathrm{O}$ $(3 \mathrm{HT})_{15}$-COOtBu (measured mass: $2753.3833 \mathrm{u} /$ calculated mass : $2753.3812 \mathrm{u} / 0.8 \mathrm{ppm}$ error). This suggests that a reaction with THF occurs. McNeil and coworkers also observed such reaction in the PPP polymerization. ${ }^{39}$ In order to find out whether this reaction occurs at the stage of the 
monomer formation or during the actual polymerization, a ${ }^{1} \mathrm{H}-\mathrm{NMR}$ spectrum was recorded of the zincated compound, quenched with $\mathrm{D}_{2} \mathrm{O}$ (see SI, Figure S27). No signs of reaction with THF were observed, demonstrating that this reaction takes place during the polymerization, terminating the polymerization. The ${ }^{1} \mathrm{H}-\mathrm{NMR}$ spectrum of the polymer, however, does not contain signals matching the THF end group, while those corresponding to the initiator moiety are clearly present.
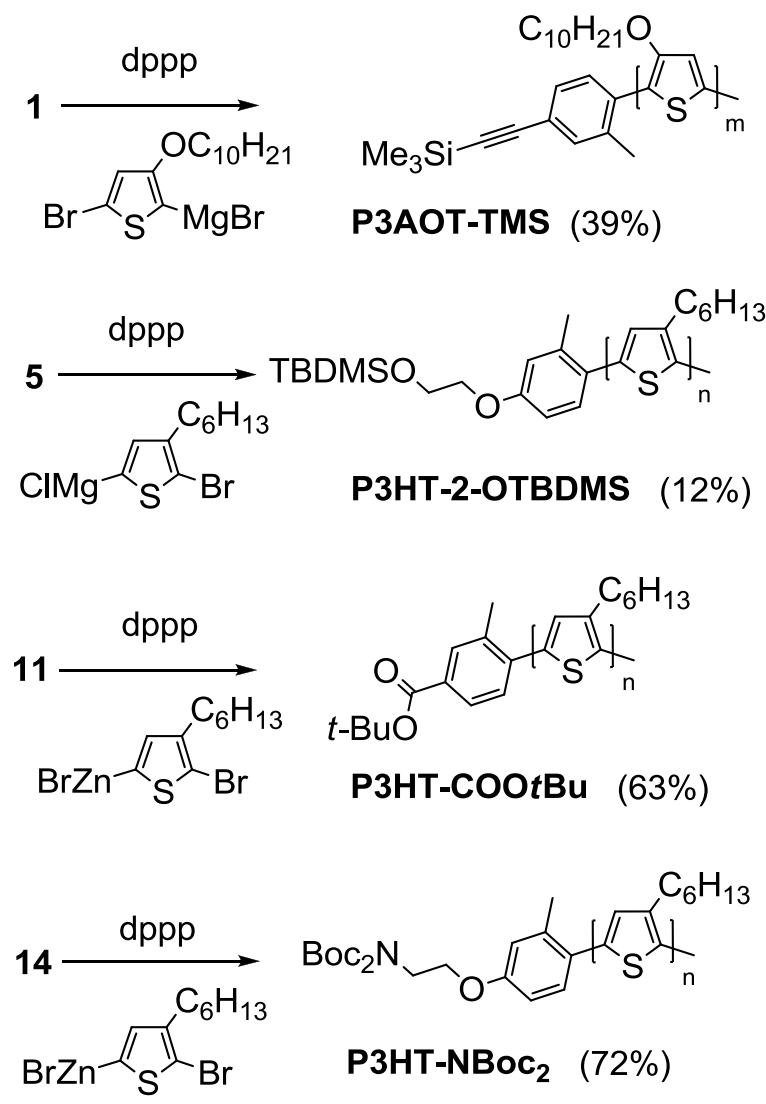

Scheme 2. Synthesis of the polymers.

Finally, the amine-functionalized P3HT was also synthesized using 2-bromozincio-4-hexyl-5bromothiophene, which was added to a solution of $\mathbf{1 4}$, after the latter underwent ligand exchange with dppp, forming P3HT-NBoc 2 . The ${ }^{1} \mathrm{H}-\mathrm{NMR}$ spectrum of P3HT-NBoc 2 , contains two methyl signals from the $o$-methyl moiety, indicating partial deprotection during polymerization or work-up. This could also be confirmed by recording the MALDI mass spectrum of the P3HT-NBoc sample $^{2}$ (see Figure S40). Indeed, this spectrum features signals corresponding to both the bis-protected polymer chains and the mono-protected molecules, respectively P3HT-NBoc 2 and P3HT-NHBoc. Nevertheless, since isobutene losses are detected in the MALDI spectrum, we cannot rule out that 
the ions corresponding to P3HT-NHBoc can also be generated by decompositions upon MALDI analysis of P3HT-NBoc 2 molecules into P3HT-NHBoc molecules. In addition, as already observed when analyzing the P3HT-COOtBu sample, polymer ions bearing a $\mathrm{C}_{4} \mathrm{H}_{7} \mathrm{O}$ residue as end group dominate the MALDI mass spectrum.

The molar masses and polydispersities were determined by GPC calibrated towards polystyrene standards. The DP of the P3HTs was also determined by ${ }^{1} \mathrm{H}-\mathrm{NMR}$ integration. For P3HT-2OTBDMS, it was found to be equal to 10, which correlates well with the GPC result. For P3HTCOOtBu (26) and P3HT-NBoc 2 (27) significantly higher than expected molar masses were found, which suggests an inefficient initiation by the respective Ni-initiators. The polydispersity, however, remains low. GPC, on the other hand, gives higher values. It should be noted that GPC tends to overestimate the molecular weight of P3HTs and that this error increases with higher molar mass. ${ }^{40}$ This effect is also observed for P3AOTs which can be explained by the more rigid conformation of P3AOTs in THF, which explains the relatively high value for P3AOT-TMS. Its higher polydispersity can be attributed to the fact that the polymerization is not controlled.

Table 1. $\bar{M}$ n and PDI for the homopolymers determined by GPC calibrated towards polystyrene standards in THF.

\begin{tabular}{|l|l|l|}
\hline Polymer & $\begin{array}{l}\bar{M}_{\mathrm{n}} \\
(\mathrm{kg} / \mathrm{mol})\end{array}$ & \\
\hline P3HT-2-OTBDMS & 1,9 & 1,3 \\
\hline P3AOT-TMS & 8,1 & 1,6 \\
\hline P3HT-COOtBu & 7,1 & 1,2 \\
\hline P3HT-NBoc $_{2}$ & 7,3 & 1,2 \\
\hline
\end{tabular}

\section{Post-polymerization transformations}

The next step was the transformation of the prepared polymers to those with the initially desired functional group, either through deprotection or conversion to another functionality (Scheme 3). For 
P3HT-2-OTBDMS, in addition to the deprotection reaction, conversion to the azide was required. Deprotection was achieved by reaction with TBAF, which furnished the alcohol-functionalized polymer P3HT-2-OH. Next, the alcohol functionality in P3HT-2-OH had to be converted into an azide. We wondered whether it was possible to achieve direct conversion of the alcohol to the azide, in order to limit the number of post-polymerization reactions, as the removal of remaining reactants from the resulting polymer is not always straightforward and can be detrimental for the final yield. Subsequent literature study revealed that diphenylphosphorylazide (DPPA) is able to achieve quantitative conversion of alcohols to azides in small molecule synthesis, using mild conditions. ${ }^{41}$ We found that, when reacting P3HT-2-OH with DPPA in the presence of DBU, a substantial shift in ppm value for the ethyl protons is observed, as expected. However, the IR spectrum of the resulting P3HT did not contain the expected azide peak around $2100 \mathrm{~cm}^{-1}$. Careful examination of the aromatic region of the ${ }^{1} \mathrm{H}-\mathrm{NMR}$ spectrum showed that the reaction stopped at the stage of the diphenyl phoshonate. We were therefore forced to shift our approach to a two-step conversion, first converting P3HT-2-OH with $p$-toluenesulfonylchloride and $\mathrm{NaOH}$ to the tosylate P3HT-O-Ts, which was isolated and thereafter converted to the azide functionalized polymer (P3HT-2-N $\mathbf{3})$ by reaction with tetrabutylammonium azide. Although the pathway through the tosylate requires an additional step, there is also a benefit related with it, because the tosylate moiety in itself is an interesting functionality as it can serve as a springboard for many other functional groups besides the azide. 


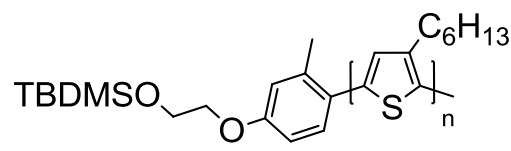

P3HT-2-OTBDMS

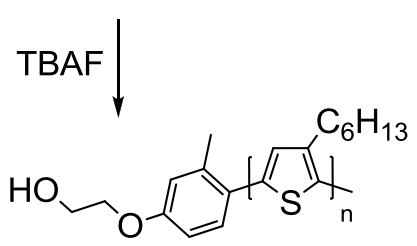

P3HT-2-OH

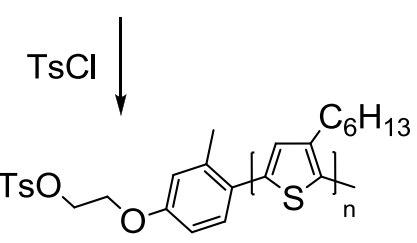

P3HT-2-OTs

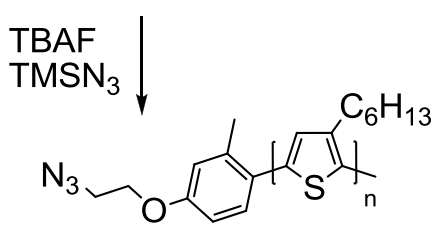

P3HT-2-N 3

Scheme 3. Post-polymerization deprotection of P3HT-2-OTBDMS and conversion to P3HT-2-N . $_{\text {. }}$

Analysis of P3HT-2-N 3 by IR spectroscopy (absorption at $2103 \mathrm{~cm}^{-1}$, see Figure S50) and MALDI-ToF confirmed the successful insertion of the azide. In the MALDI mass spectrum, presented in Figure S41, signals corresponding to ionized P3HT end capped with a hydrogen atom and the azide-containing group were clearly identified. In addition, a characteristic nitrogen loss was observed and definitively confirmed the presence of the azide moiety. The presence of P3HT polymer chains with $\mathrm{H} / \mathrm{H}$ as the end groups was also highlighted by the MALDI measurement, which is due to the fact that they were already present for P3HT-2-OTBDMS and are not affected by the post-polymerization reactions. As a final confirmation, the different conversion steps of P3HT-2-OTBDMS to P3HT-2-N $\mathbf{N}_{3}$ can all be monitored by observing the chemical shifts of the ethyl protons, as illustrated in Figure 2. 


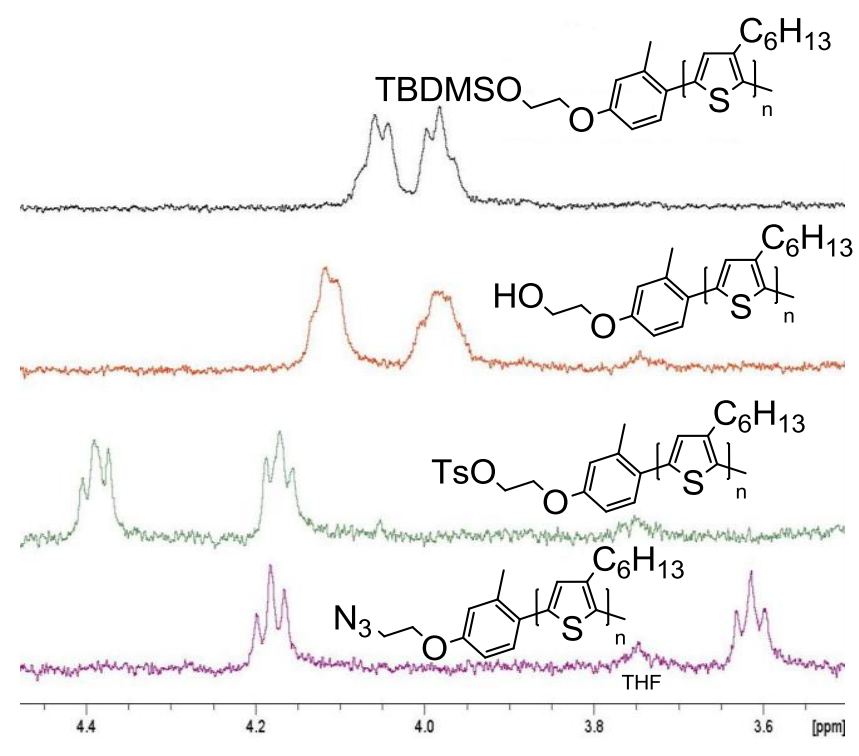

Figure 2. Chemical shift of the ethyl protons in function of the end group in the postpolymerization reactions of P3HT-2-OTBDMS.

In the case of P3AOT-TMS, the TMS moiety was removed using TBAF, yielding the ethynylene functionalized polymer P3AOT-E. Again deprotection was confirmed both by ${ }^{1} \mathrm{H}-\mathrm{NMR}$ and MALDI-ToF (see Figure S42). As already observed when analyzing the protected molecules (P3AOT-TMS), the MALDI process also leads to the loss of a $\mathrm{C}_{10} \mathrm{H}_{21}$ radical from the ionized P3AOT-E polymers.

The deprotection of P3HT-NBoc 2 proved to be unexpectedly difficult, as treatment with an excess of trifluoroacetic acid only achieved partial deprotection to the mono-protected amine. Substituting TFA for $p$-toluenesulfonic acid and performing the reaction under reflux conditions for $48 \mathrm{~h}$, finally, yielded P3HT-NH 2 , as indicated by the shift of the ethyl protons in the ${ }^{1} \mathrm{H}-\mathrm{NMR}$ spectrum (Figure 3). This was also confirmed by the MALDI-ToF analysis (see Figure S43). 


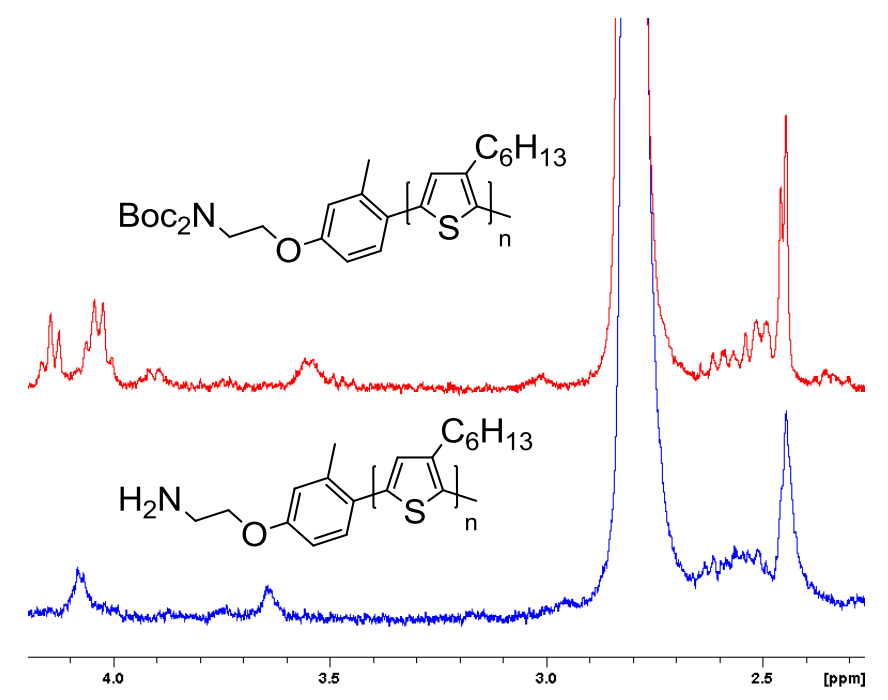

Figure 3. Chemical shift of the ethyl protons in the deprotection of P3HT-NBoc 2 .

Finally, P3HT-COOtBu was deprotected to P3HT-COOH. Initial attempts employing KOH were not successful. Fortunately, the use of $\mathrm{NBu}_{4} \mathrm{OH}$ readily resulted in a clean and quantitative deprotection, as shown by the MALDI-ToF spectra (see Figure S44) and IR spectroscopy, which show a shift from $1713 \mathrm{~cm}^{-1}$ (ester carbonyl) to $1684 \mathrm{~cm}^{-1}$ (carboxylic acid carbonyl) (see Figures S47-50).

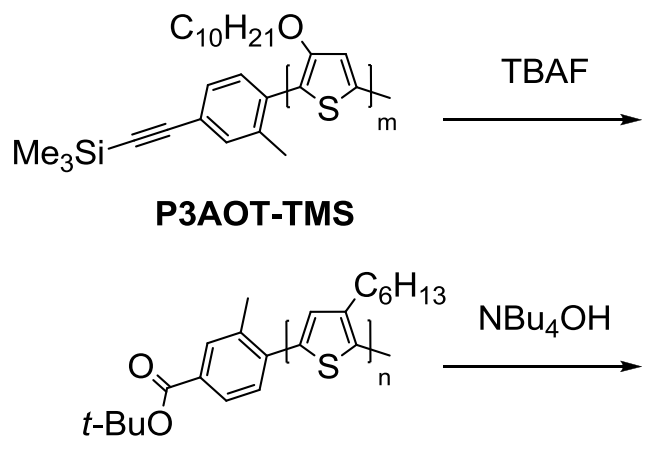

P3HT-COOtBu

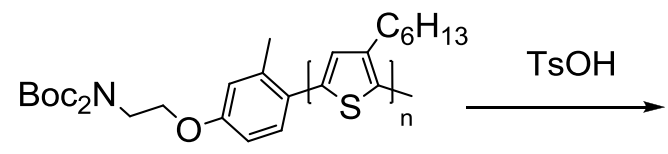

P3HT-NBoc 2

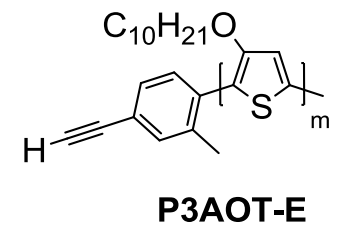<smiles>CC(C)=C(C)Sc1ccc(C(=O)O)cc1C</smiles>

P3HT-COOH

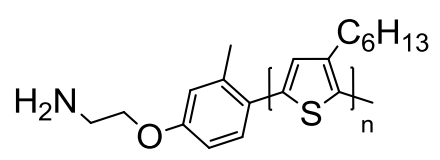

P3HT- $\mathrm{NH}_{2}$

Scheme 4. Post-polymerization deprotection of the polymers. 


\section{Block-copolymer formation}

The successful synthesis of P3AOT-E and P3HT-2-N 3 provided us with two polymeric substrates suitable for application in CuAAC click-chemistry. As both polymers are only soluble in either chloroform or THF, the standard $\mathrm{CuSO}_{4}$-ascorbic acid protocol could not be used. Instead we used a combination of $\mathrm{CuBr}$ and $N, N, N$, $N$ ', $N$ '-pentamethyldiethylenetriamine (PMDTA) in $\mathrm{THF},{ }^{42}$ with a slight excess of P3HT-2-N $\mathbf{N}_{3}$ to perform the click reaction, resulting in the formation of P3HT-2-

\section{P3AOT.}

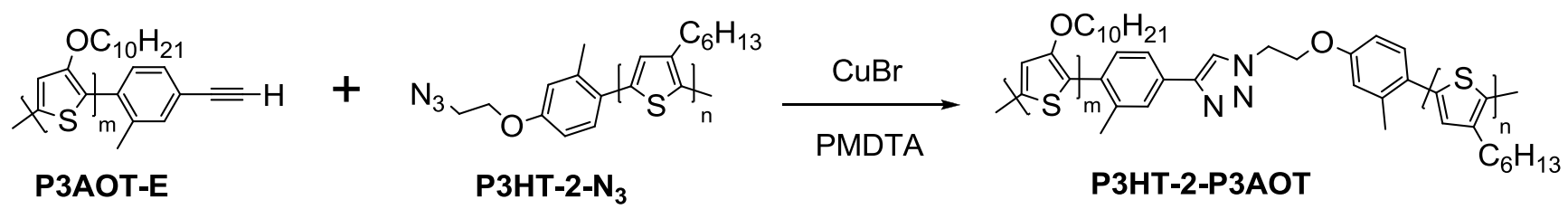

Scheme 5. Block-copolymer formation.

In order to remove unreacted homopolymers, the crude P3HT-2-P3AOT was successively fractionated with pentane, hexane, heptane and chloroform, with the P3HT-2-P3AOT being dissolved in the latter fraction. GPC analysis of this fraction (Figure 4) showed a shift to higher molar mass compared to both P3AOT-E and P3HT-2-N $\mathbf{N}_{\mathbf{3}}$, confirming the formation of blockcopolymer while excluding the possibility of a blend of the two homopolymers. ${ }^{1} \mathrm{H}-\mathrm{NMR}$ analysis clearly shows the $\alpha$-methylene signals for the P3AOT $(4.21 \mathrm{ppm})$ and the P3HT $(2.78 \mathrm{ppm})$ block. From the integration of these signals it can also be deduced that the ratio P3AOT:P3HT is 4:1.

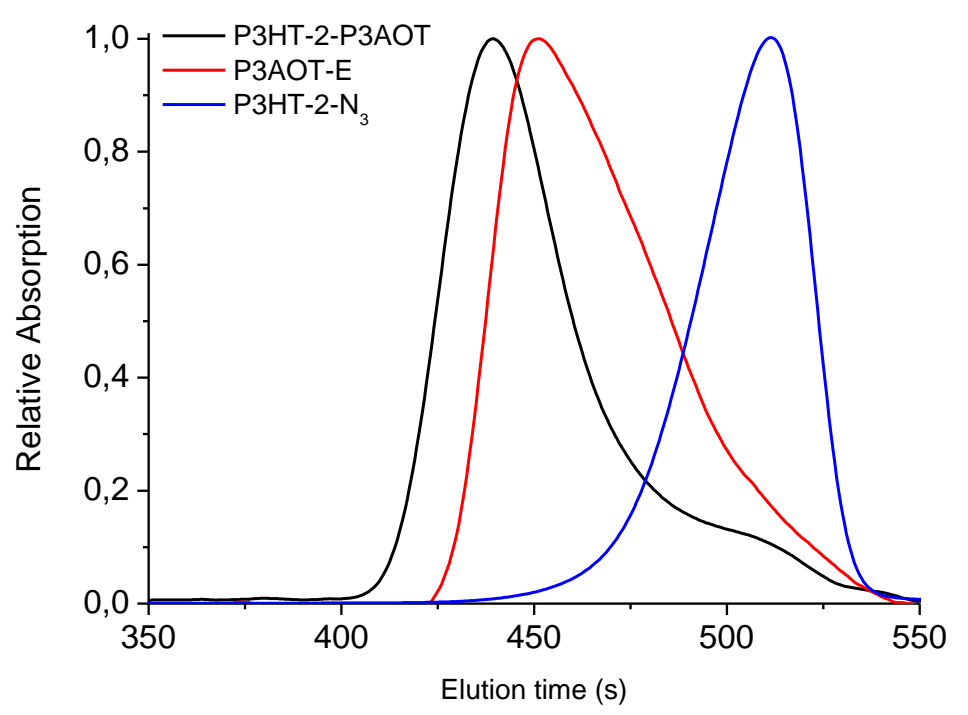

Figure 4. GPC elution profile for P3HT-2-P3AOT compared with its constituting homopolymers 


\section{Bifunctional Ni-initiator}

Recent research ${ }^{43}$ suggests that during the polymerization of P3HT using a Ni-catalyst, ring walking of the Ni-center allows the polymerization to proceed from both chain ends. However, when two significantly different monomers are polymerized, propagation can be expected to occur only at one side, since the complexation of the Ni-moiety is more favored for one system than to the other one and the Ni-catalysts cannot cross this barrier. We therefore decided to investigate whether it would be possible to grow P3HT simultaneously from both chain ends, using two nickel atoms propagating at the same time in opposite directions. The experimental verification of this hypothesis could be achieved by initiating the polymerization of P3HT with an initiator carrying two nickel atoms. In order to mimic the properties of a P3HT chain, we decided to use a bithiophene moiety, with methyl substituents ortho to the nickel moiety for stability reasons. Therefore, 5,5'-dibromo4,4'-dimethyl-2,2'-bithiophene (17) was prepared through palladium coupling of 2-bromo-3methylthiophene ${ }^{44}(\mathbf{1 6})$, which was in turn prepared by bromination of 3-methylthiophene (15). Subsequently, 17 was allowed to react with two equivalents of $\mathrm{Ni}\left(\mathrm{PPh}_{3}\right)_{4}$, resulting in the formation of the bifunctional nickel initiator 18. Although $\mathbf{1 8}$ was succesfully isolated, it showed extremely rapid decomposition in solution, which severely complicated its analysis and explains its low yield. However, when combined with dppp, the stability was sufficient to allow initiation of the polymerization. When 2-magnesiochloro-4-hexyl-5-bromothiophene was added to a solution of $\mathbf{1 7}$ which had first undergone ligand exchange with dppp, polymerization did occur, as expected. ${ }^{1} \mathrm{H}-$ NMR of the $\mathrm{H}^{+}$-terminated P3HT-bth-P3HT shows that polymerization did indeed proceed on both sides, as only one singlet at $2.42 \mathrm{ppm}$ is visible for both $4,4^{\prime}$-methyls and there is a clear shift from 17 (Figure 5). Also, the presence of only one triplet at $2.62 \mathrm{ppm}$, arising from the $\alpha$-methylenes from hydrogen terminated P3HT chains, ${ }^{27}$ indicates that the polymerization is controlled. The fact that there is no distinct triplet for the $\alpha$-methylenes of P3HT next to the bithiophene moiety is probably due to the high similarity of the bithiophene to the P3HT backbone. MALDI-ToF spectroscopy reveals that indeed only $\mathrm{H} / \mathrm{H}$ terminated chains are present (SI, Figure S45). GPC-analysis of P3HTbth-P3HT results in an $\bar{M}_{\mathrm{n}}$ of $3.8 \mathrm{~kg} / \mathrm{mol}$ and a PDI of 1.6 . 


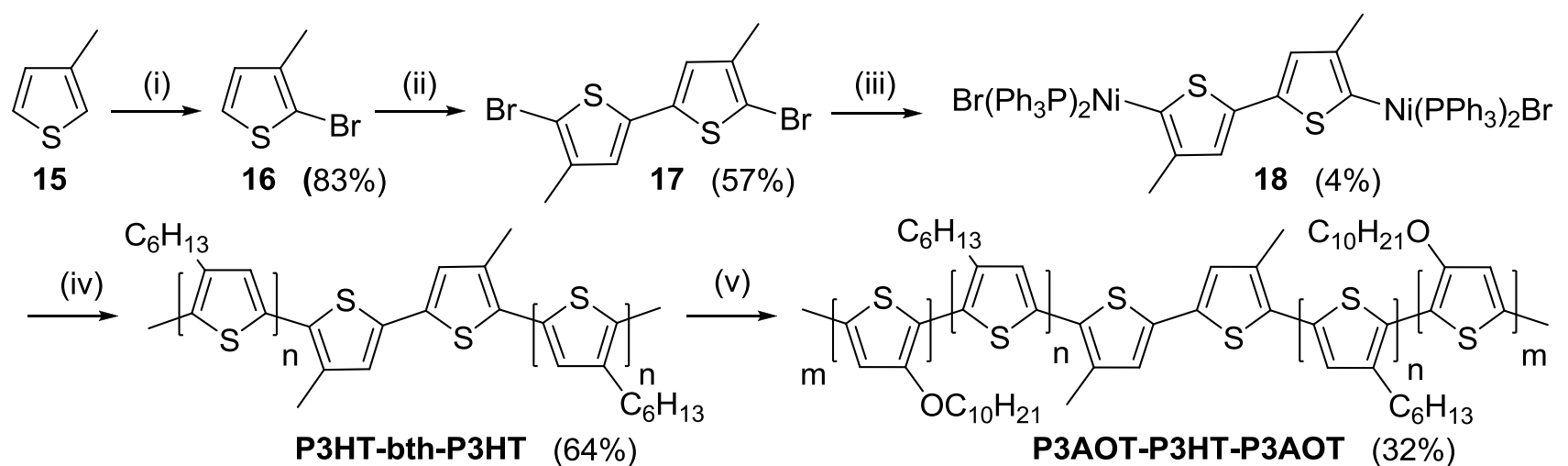

Scheme 6. Synthesis of the bifunctional nickel initiator and subsequent polymerization. (i) NBS (ii) $\mathrm{PdCl}_{2}(\mathrm{PhCN})_{2}, \quad \mathrm{AgNO}_{3}, \quad \mathrm{KF}$ (iii) $\mathrm{Ni}\left(\mathrm{PPh}_{3}\right)_{4}$ (iv) 1) dppp 2) 2-magnesiochloro-4-hexyl-5bromothiophene (v) 2-bromomagnesio-5-bromo-3-(3,7-(S)-dimethyloctyloxy)thiophene or $\mathrm{H}_{3} \mathrm{O}^{+}$

If one takes into consideration that (a) the polymerization does indeed proceed on both sides and (b) the polymerization of P3HT is controlled when employing dppp-ligated nickel catalysts, it should be possible to prepare a diblock-copolymer by extending the P3HT block on P3HT-bth-P3HT by addition of a second monomer. Therefore, after the polymerization of P3HT-bth-P3HT, one batch was quenched with acid, while 2-bromomagnesio-5-bromo-3-(3,7-(S)-dimethyloctyloxy)thiophene was added to the other batch and the polymerization was allowed to proceed further. If the conditions mentioned above are met, the latter should result in the formation of a diblock-copolymer of P3HT and P3AOT on both sides of the bithiophene moiety, in the form of P3AOT-P3HT-bthP3HT-P3AOT. Essentially, this can be regarded as a P3AOT-P3HT-P3AOT triblock-copolymer, and to the best of our knowledge, it is the first time that such a structure, comprised of entirely conjugated blocks, has been prepared by a chain-growth polycondensation. In the ${ }^{1} \mathrm{H}-\mathrm{NMR}$ spectrum, the $\alpha$-methylene protons of the P3AOT-block (4.22 ppm) and P3HT-block (2.80 ppm) have a ratio of 3.5:1. By comparing the integration of these with the singlet of the bithiophene methyls at $2.42 \mathrm{ppm}$, it can be concluded that the P3HT-block contains 7 units and the P3AOT-block 24 on each side, which corroborates the previously calculated ratio. The disappearance of the triplet at $2.62 \mathrm{ppm}$ upon addition of the second monomer, clearly demonstrates a successful initiation of the P3AOT-blocks on both sides of the P3HT-bth-P3HT. 

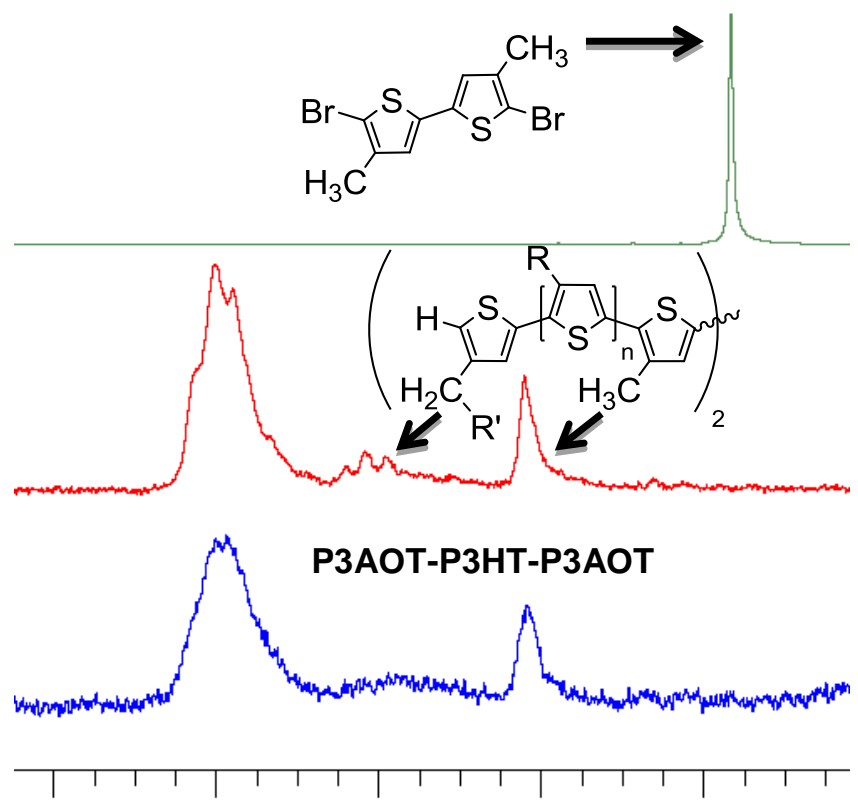

$\begin{array}{lllll}3.0 & 2.8 & 2.6 & 2.4 & {[\mathrm{ppm}]}\end{array}$

Figure 5. ${ }^{1} \mathrm{H}-\mathrm{NMR}$ comparison of $\mathbf{1 7}$ and block-copolymers.

GPC-analysis shows a clear shift to shorter elution times (and thus higher molecular weight) for P3AOT-P3HT-P3AOT compared to P3HT-bth-P3HT. In order to demonstrate that the product did not simply consist of a mixture of homopolymers, we also measured GPC elution times at $430 \mathrm{~nm}$ and $600 \mathrm{~nm}$, wich correspond to the respective absorption maxima of P3HT and P3AOT. The fact that these traces (Figure 6) all show the same elution time for P3AOT-P3HT-P3AOT proves the formation of a block-copolymer and excludes the possibility of blends.

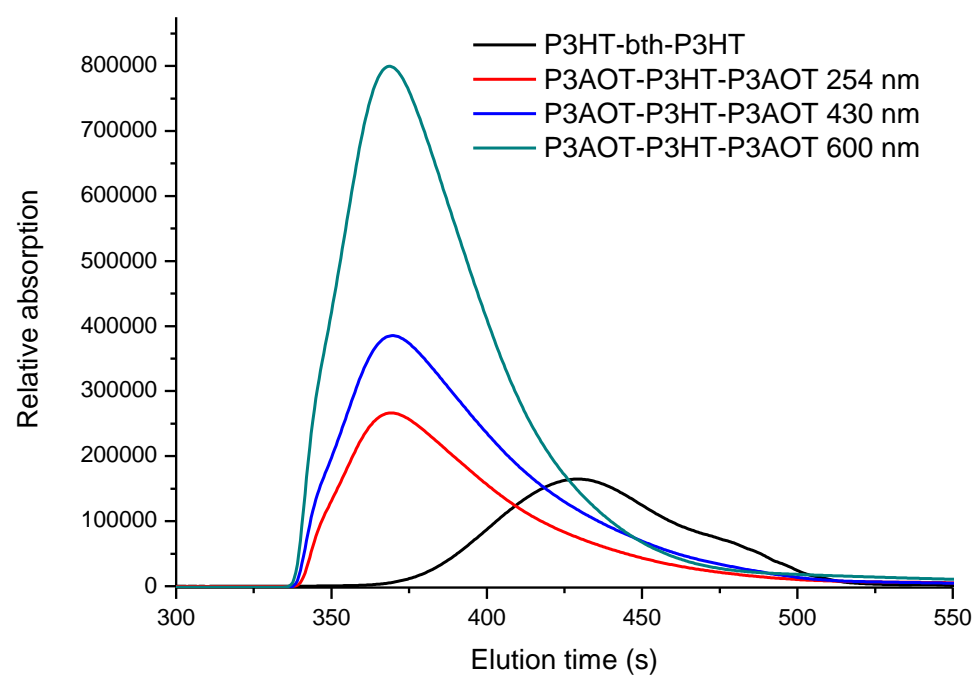

Figure 6. GPC elution profile of P3HT-bth-P3HT compared with P3AOT-b-P3HT-b-P3AOT. 


\section{Conclusion}

In conclusion, we have succeeded in preparing several polythiophenes with ethynylene, alcohol, tosylate, azide, amine and carboxylic acid functionalities. We also demonstrated that these end groups can be transformed in a straightforward manner and can be used for the synthesis of a conjugated block-copolymer with two electronically different blocks. For the first time, a bifunctional nickel initiator was synthesized and used to prepare a triblock-copolymer consisting of fully conjugated blocks.

Ackowledgments. We are grateful to the Katholieke Universiteit Leuven (GOA) and the Fund for Scientific Research (FWO-Vlaanderen) for financial support. A.F. and P.W. are doctoral fellows of IWT-Vlaanderen. P.G. and J.D.W. (FRS-FNRS research associate and research fellow) are grateful to the FRS-FNRS for financial support in the acquisition of the Waters QToF Premier mass spectrometer and for continuing support.

Supporting Information Available. Experimental procedures, ${ }^{1} \mathrm{H}-\mathrm{NMR},{ }^{13} \mathrm{C}-\mathrm{NMR},{ }^{31} \mathrm{P}-\mathrm{NMR}$, IR and MALDI-ToF of selected compounds and/or polymers. This material is available free of charge via the Internet at http://pubs.acs.org.

\section{References}

(1) Krebs, F. C.; Senkovskyy, V.; Kiriy A. IEEE J. Sel. Topics Quant. Electronics 2010, 16, 18211826.

(2) Iovu, M. C.; Sheina, E. E.; Gil, R. R.; McCullough, R. D. Macromolecules 2005, 38, 86498656.

(3) Sheina, E. E.; Liu, J.; Iovu, M. C.; Laird, D. W.; McCullough, R. D. Macromolecules 2004, 37, 3526-3528.

(4) Miyakoshi, R.; Yokoyama, A.; Yokozawa, T. J. Am. Chem. Soc. 2005, 127, 17542-17547.

(5) Yokoyama, A.; Miyakoshi, R.; Yokozawa, T. Macromolecules 2004, 37, 1169-1171. 
(6) Iovu, M. C.; Jeffries-EL, M.; Sheina, E. E.; Cooper, J. R.; McCullough, R. D. Polymer 2005, $46,8582-8586$.

(7) Iovu, M. C.; Craley, C. R.; Jeffries-EL, M.; Krankowski, A. B.; Zhang, R.; Kowalewski, T.; McCullough R. D. Macromolecules 2007, 40, 4733-4735.

(8) Liu, J.; Sheina, E.; Kowalewski, T.; McCullough, R. D. Angew. Chem. Int. Ed. 2002, 41, 329332.

(9) Yokozawa, T.; Adachi, I.; Miyakoshi, R.; Yokoyama, A. High Performance Polymers 2007, 19, 684-699.

(10) Wu, P.; Ren, G.; Li, C.; Mezzenga, R.; Jenekhe, S. A. Macromolecules 2009, 42, 2317-2320.

(11) Ouhib, F.; Khoukh, A.; Ledeuil, J.; Martinez, J.; Desbières, J.; Dagron-Lartigau, C. Macromolecules 2008, 41, 9736-9743.

(12) Ohshimizu, K.; Ueda, M. Macromolecules 2008, 41, 5289-5294.

(13) Zhang, Y.; Tajima, K.; Hirota, K.; Hashimoto, K. J. Am. Chem. Soc 2008, 130, 7812-7813.

(14) Zhang, Y.; Tajima, K.; Hashimoto, K. Macromolecules 2009, 42, 7008-7015.

(15) Van den Bergh, K.; Huybrechts, J.; Verbiest, T.; Koeckelberghs, G. Chem. - Eur. J. 2008, 14, $9122-9125$.

(16) Van den Bergh, K.; Cosemans, I.; Verbiest, T.; Koeckelberghs, G. Macromolecules 2010, 43, 3794-3800.

(17) Hollinger, J.; Jahnke, A. A.; Coombs, N.; Seferos, D. S. J. Am. Chem. Soc. 2010, 132, 85468547.

(18) Javier, A. E.; Varshney, S. R.; McCullough, R. D. Macromolecules 2010, 43, 3233-3237. 
(19) Yokoyama, A.; Rato, A.; Miyakoshi, R.; Yokozawa, T. Macromolecules 2008, 41, 72717273.

(20) Huang, L.; Wu, S.; Qu, Y.; Geng, Y.; Wang, F. Macromolecules 2008, 41, 8944-8947.

(21) Miyakoshi, R.; Yokoyama, A.; Yokozawa, T. J. Polym. Sci. Part A: Polym. Chem. 2008, 46, 753-765.

(22) Beryozkina, T.; Boyko, K.; Khanduyeva, N.; Senkovskyy, V.; Horecha, M.; Oertel, U.; Simon, F.; Stamm, M.; Kiriy, A. Angew. Chem. Int. Ed. 2009, 48, 2695-2698.

(23) Yokozawa, T.; Kohno, H.; Ohta, Y.; Yokoyama, A. Macromolecules 2010, 43, 7095-7100.

(24) Bronstein, H. A.; Luscombe, C. K. J. Am. Chem. Soc. 2009, 131, 12894-12895.

(25) Doubina, N.; Stoddard, M.; Bronstein, H. A.; Jen, A. K.-Y.; Luscombe, C. K. Macromol. Chem. Phys. 2009, 210, 1966-1972.

(26) Doubina, N.; Ho, A.; Jen, A. K.-Y.; Luscombe, C. K. Macromolecules 2009, 42, $7670-7677$.

(27) Senkovskyy, V.; Khanduyeva, N.; Komber, H.; Oertel, U.; Stamm, M.; Kuckling, D.; Kiriy, A. J. Am. Chem. Soc. 2007, 129, 6626-6632.

(28) Senkovskyy, V.; Tkachov, R.; Beryozkina, T.; Komber, H.; Oertel, U.; Horecha, M.; Bocharova, V.; Stamm, M.; Gevorgyan, S. A.; Krebs, F. C.; Kiriy, A. J. Am. Chem. Soc. 2009, 131, 16445-16453.

(29) Kaul, E.; Senkovskyy, V.; Tkachov, R.; Bocharova, V.; Komber, H.; Stamm, M.; Kiriy, A. Macromolecules 2010, 43, 77-81.

(30) Smeets, A.; Van den Bergh, K.; De Winter, J.; Gerbaux, P.; Verbiest, T.; Koeckelberghs G. Macromolecules 2009, 42, 7638-7641.

(31) Meldal, M.; Tornøe, C. W. Chem. Rev. 2008, 108, 2952-3015. 
(32) Sharpless, K. B.; Finn, M. G.; Kolb H. C. Angew. Chem. Int. Ed. 2001, 40, 2004-2021.

(33) Liu, J.; Tanaka, T.; Sivula, K.; Alivisatos, A. P.; Frechet, J. M. J. J. Am. Chem. Soc. 2004, $126,6550-6551$.

(34) Lohwasser, R.; Bandara, J.; Thelakkat, M. J. Mater. Chem., 2009, 19, 4126-4130.

(35) Van den Bergh, K.; De Winter, J.; Gerbaux, P.; Verbiest, T.; Koeckelberghs G. Macromol. Chem. Phys. 2010, 212, 328-335.

(36) Chen, T.-A.; Wu, X.; Rieke, R. D. J. Am. Chem. Soc. 1995, 117, 233-244.

(37) Doubina, A.; Paniagua, S. A.; Soldatova, A. V.; K. Y. Jen, A.; Marder, S. R.; Luscombe, C. K. Macromolecules 2011, 44, 512-520.

(38) De Winter, J.; Deshayes, G.; Boon, F.; Coulembier, O.; Dubois, P.; Gerbaux, P. J. Mass. Spectrom. 2011, 46, 237-246.

(39) Lanni, E. L.; McNeil, A. J. J. Am. Chem. Soc. 2009, 131, 16573-16579.

(40) Liu, J.; Loewe, R. S.; McCullough, R. D. Macromolecules 1999, 32, 5777-5785.

(41) Thompson, A. S.; Humphrey, G. R.; DeMarco, A. M.; Mathre, D. J.; Grabowski, E. J. J. J. Org. Chem. 1993, 58, 5886-5888.

(42) Urien, M.; Erothu, H.; Cloutet, E.; Hiorns, R. C.; Vignau, L.;Cramail, H. Macromolecules 2008, 41, 7033-7040.

(43) Tkachov, R.; Senkovskyy, V.; Komber, H.; Sommer, J.-U.; Kiriy, A.; J. Am. Chem. Soc. 2010, $132,7803-7810$.

(44) Takahashi, M.; Masui, K.; Sekiguchi, H.; Kobayashi, N.; Mori, A.; Funahashi, M.; Tamaoki, N. J. Am. Chem. Soc. 2006, 128, 10930-10933. 


\section{For Table of Contents use only}

End Group-functionalization and Synthesis of Block-copolythiophenes by Modified Nickel Initiators

Alfons Smeets, Pieter Willot, Julien De Winter, Pascal Gerbaux, Thierry Verbiest and Guy Koeckelberghs
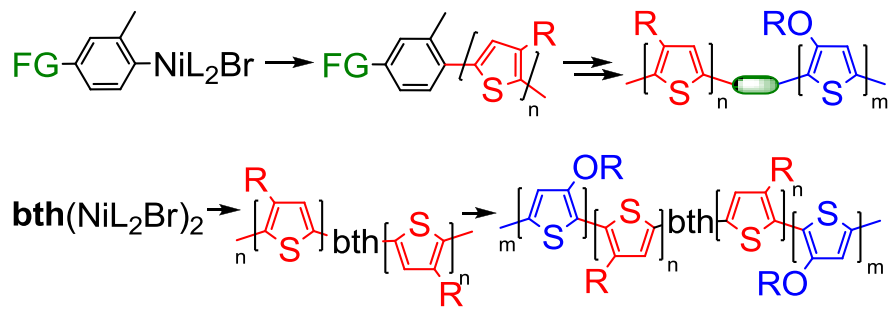